\title{
Comparison of fluorescent in situ hybridization HER-2/neu results on core needle biopsy and excisional biopsy in primary breast cancer
}

\author{
Sophia K Apple, Alarice C Lowe, P Nagesh Rao, I Peter Shintaku and Neda A Moatamed
}

Department of Pathology and Laboratory Medicine, David Geffen School of Medicine at UCLA, UCLA Center for the Health Sciences, Los Angeles, CA, USA

\begin{abstract}
HER-2/neu status is critical for the therapy for breast carcinoma. Fluorescent in situ hybridization for gene amplification and immunohistochemical stains for protein expression are widely used methods to detect HER-2/neu status. Multiple studies have shown fluorescent in situ hybridization and immunohistochemical stain results to have high concordance rates. To our knowledge, a comparison between fluorescent in situ hybridization results for core needle biopsy and the subsequent excisional biopsy specimens has not yet been studied. We retrospectively evaluated the fluorescence in situ hybridization and immunohistochemical results in both the breast core needle and the excisional biopsy of 125 patients with invasive breast carcinoma from 2002 to 2005. There was complete concordance with respect to both immunohistochemical and fluorescence in situ hybridization results for core needle biopsy and excisional biopsy specimens in $87 \%$ of the patients evaluated. Comparison of fluorescent in situ hybridization results of the 129 core needle biopsies to the 131 excisional biopsies of all 125 patients showed a concordance rate of $92 \%$. The immunohistochemical stain results of the same core needle and excisional biopsies showed a concordance rate of $98 \%$. Comparison of the immunohistochemical stain results with the fluorescent in situ hybridization results for all 260 cases examined showed $95 \%$ concordance. On the basis of our study, we observed that repeating HER-2/neu testing by immunohistochemical stain and/or fluorescent in situ hybridization methods on excisional biopsy is not unreasonable, in particular in cases of intratumoral heterogeneity, indeterminate/borderline $H E R-2 /$ neu results and after neoadjuvant chemotherapy.

Modern Pathology (2009) 22, 1151-1159; doi:10.1038/modpathol.2009.82; published online 29 May 2009
\end{abstract}

Keywords: HERr-2/neu; fluorescent in situ hybridization; immunohistochemical stains; core needle biopsy; excisional biopsy

Determination of tumor HER-2/neu receptor overexpression/amplification status is one of the most important clinical assessments in the standard workup for breast carcinoma because its presence warrants the addition of trastuzumab (Herceptin) to the patient's medical oncology regimen.

HER-2/neu testing to evaluate for trastuzumab therapy is a prototypical example of targeted treatment. Currently, there are two widely used methods for determination of $H E R-2 / n e u$ status, fluorescent

Correspondence: Dr SK Apple, MD, Pathology and Laboratory Medicine, David Geffen School of Medicine at UCLA, UCLA Center for the Health Sciences, 10833 Le Conte Ave, CHS, Los Angeles, CA 90095, USA.

E-mail: sapple@mednet.ucla.edu

Received 5 March 2009; revised and accepted 5 May 2009; published online 29 May 2009 in situ hybridization for the gene amplification and immunohistochemical studies for protein overexpression. Common practice is to perform immunohistochemical stains on all breast cancer specimens and subsequently perform fluorescent in situ hybridization only on specimens with an HER-2/neu immunohistochemical stain score of 2 (on a scale of 0-3). At institutions following this protocol, fluorescent in situ hybridization is not performed on specimens with immunohistochemical scores of 0 or 1 , which are interpreted as no overexpression of the HER2/neu receptor, or with an immunohistochemical score of 3 , which is interpreted as overexpression of the HER-2/neu receptor. Also, HER-2/neu status is often performed on either the core needle or the excisional biopsy specimen and usually not on both. The core needle biopsy sample is often used to study breast biomarkers when neoadjuvant chemotherapy is 
planned. To the best of our knowledge, no study has compared immunohistochemical stains and fluorescent in situ hybridization of HER-2/neu results on the core needle biopsy and excisional biopsy from the same patient. We sought to determine the correlation between HER-2/neu fluorescent in situ hybridization and immunohistochemical stain results on core needle biopsy and the corresponding excisional biopsy on a selected patient population.

In 2007, the American Society of Clinical Oncology/College of American Pathologists (ASCO/CAP) revised the guidelines for the evaluation of HER-2/ neu overexpression by immunohistochemical stain in an attempt to optimize the accuracy of HER-2/neu testing in breast cancer, based on the notion that approximately $20 \%$ of immunohistochemical stain test results from community-based laboratories may have been inaccurate when compared to high-volume reference laboratories. ${ }^{1}$ In addition, the guidelines for the evaluation of $H E R-2 / n e u$ amplification by fluorescent in situ hybridization have been updated, incorporating a 'borderline' category (fluorescent in situ hybridization ratios $\geq 1.8$ and $\leq 2.2$ ) that is neither amplified nor not amplified.

After review of the correlation data, discrepant cases were identified from our study. Three pathologists blindly re-reviewed these cases to try to rectify these discrepancies. We re-reviewed the HER-2/neu immunohistochemical stain slides of the cases with immunohistochemical stain/fluorescent in situ hybridization discrepancies, core needle biopsy/excisional biopsy discrepancies, and/or borderline fluorescent in situ hybridization ratios using ASCO/CAP 2007 guidelines to see if the new guidelines may provide more consistent results.

\section{Materials and methods}

\section{Sample Collection}

A total of 125 patients with invasive carcinoma of the breast present in both the breast core needle biopsy and the subsequent excisional biopsy (lumpectomy or mastectomy) were identified from 2002 to 2005 at our institution, including 5 patients who received neoadjuvant chemotherapy. A standard breast biomarker panel was performed on each specimen, composed of immunohistochemical stains for estrogen receptor, progesterone receptor, Ki-67 proliferative index, and HER-2/neu receptor as well as fluorescent in situ hybridization for HER-2/neu amplification. This panel was ordered reflexively on all core needle biopsy and on the excisional biopsy block containing the largest amount of invasive carcinoma. The immunohistochemical stains were evaluated by the attending pathologists within 2-3 days of ordering the breast biomarkers panel. Fluorescent in situ hybridization analysis was ordered on all cases before evaluation of the immunohistochemical stain and performed

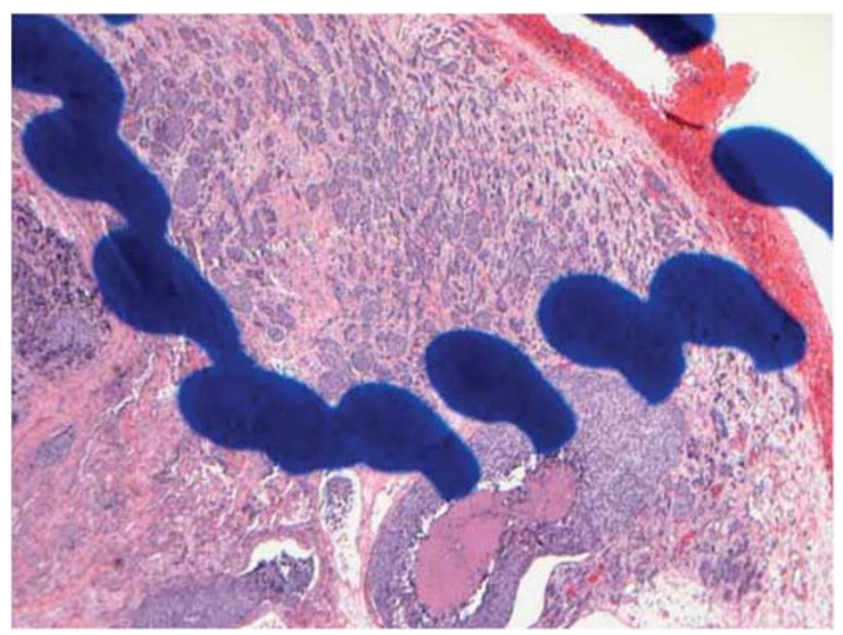

Figure $1 \mathrm{H} \& \mathrm{E}$ slide with invasive carcinoma designated by the attending pathologist.

regardless of and independent of the corresponding immunohistochemical stain result. The fluorescent in situ hybridization analysis was signed out by the Cytogenetics laboratory at our institution approximately 2 weeks after the immunohistochemical stain scores were reported. At our institution, HER-2/neu tests are carried out using the FDA approved HercepTest kit for immunohistochemical stain and Vysis PathVysion for fluorescent in situ hybridization.

\section{Immunohistochemical Stain for HER-2/neu Using HercepTest}

Paraffin sections were cut at $4 \mu \mathrm{m}$ thickness and baked for $1 \mathrm{~h}$ at $60^{\circ} \mathrm{C}$. Immunohistochemistry was performed using the HercepTest kit (Dako Corp., Carpinteria, CA, USA) according to kit instructions with positive and negative control slides included in each run.

Areas of invasive carcinoma were assessed by the attending pathologists and scored on a scale of $0-3$ according to the original FDA approved guidelines before ASCO/CAP 2007.

\section{HER-2/neu Fluorescent In Situ Hybridization Using Abbott-Vysis PathVysion}

An H\&E slide and four unstained paraffin slides were cut from the core needle biopsy block and from the block of tumor representing the largest volume of invasive carcinoma from the excisional biopsy. Two of the unstained slides were cut at a $4 \mu \mathrm{m}$ thickness. The invasive component of the carcinoma that did not include ductal carcinoma in situ (DCIS) was designated on the H\&E slide by the attending pathologist (Figure 1). The corresponding area with invasive carcinoma was studied with the fluorescent in situ hybridization protocol. Dual-color fluores- 


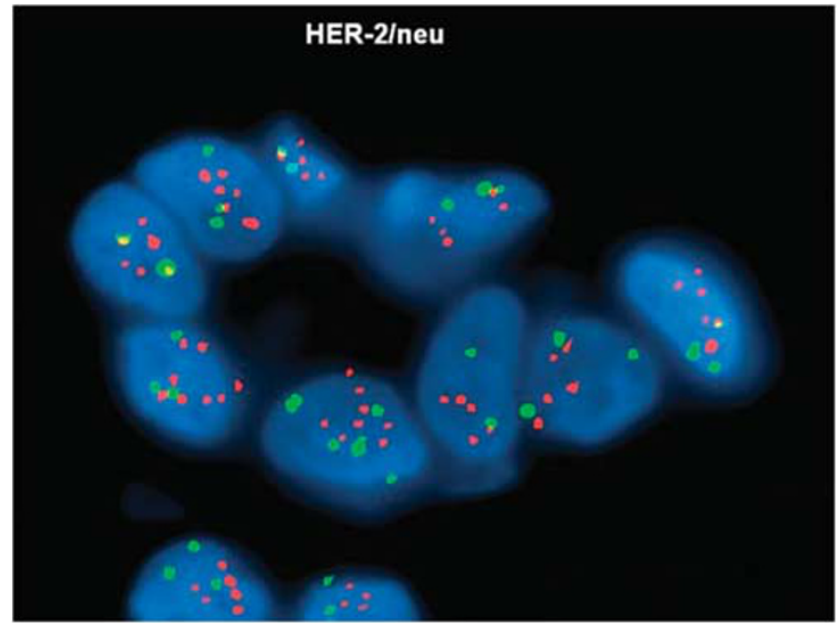

Figure 2 Dual-color FISH with HER-2 (red) and chromosome 17 centromere (green) probes with no amplification.

cent in situ hybridization was performed with the HER-2 probe labeled with spectrum red and chromosome 17 specific centromere (D17Z1) probe labeled with spectrum green on sections cut from the same block. Deparaffinization, in situ hybridization, and staining were performed using the PathVysion kit (Abbott-Vysis Lab, Abbott Park, IL, USA) as per the manufacturer's protocols. Fluorescent signals in at least 60 nonoverlapping interphase nuclei with intact morphology were scored using a Zeiss Axioplan 2 microscope with a $\times 100$ planar objective, using a triple band-pass filter that permits simultaneous blue, green, and red colors. Only tumor cells from the site designated on the H\&E slide by the pathologist were scored for the number of red (HER-2) and green (chromosome 17) signals. As recommended by the FDA-approved PathVysion HER-2 kit at the time of original interpretation, a case was scored as amplified if the ratio of the number of fluorescent signals of HER-2 to chromosome 17 (R/G) was $\geq 2.0$ (Figures 2 and 3 ).

\section{HER-2/neu Immunohistochemical Stain Slide Re-Review}

After the results were tabulated, we selected 18 patients with 36 corresponding core needle biopsy or excisional biopsy cases with discrepant HER-2/ neu results and/or a borderline fluorescent in situ hybridization ratio (defined as a fluorescent in situ hybridization ratio $\geq 1.8$ and $\leq 2.2$ ) for re-review. Six cases were unavailable because the slides were returned to the original institution; therefore, 30 cases were re-reviewed. Cases were determined to be discrepant if the fluorescent in situ hybridization results, immunohistochemical stain results, or both were inconsistent for the core needle biopsy or the excisional biopsy. Also, cases with immunohistochemical stain results that did not concur with the fluorescent in situ hybridization results were iden-

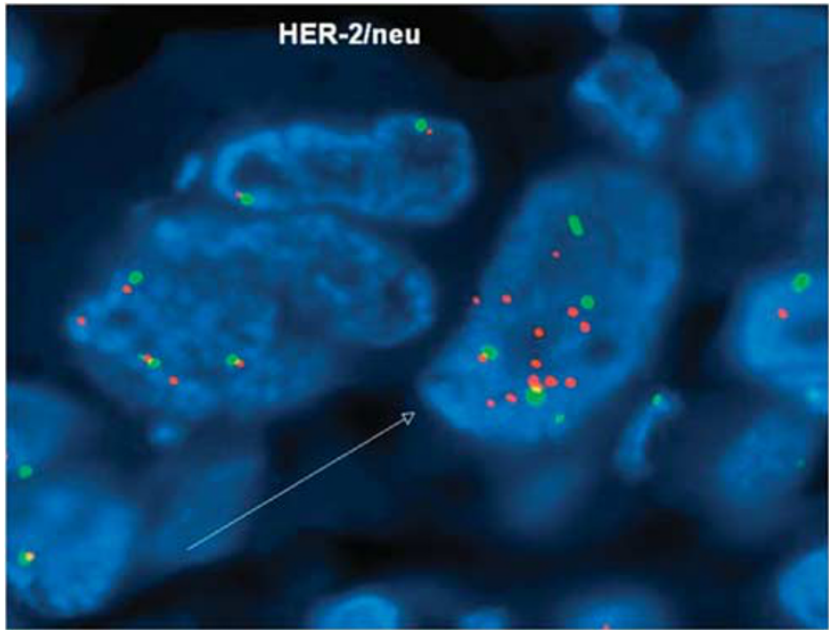

Figure 3 Dual-color FISH with HER-2 (red) and chromosome 17 centromere (green) probes with amplification.

tified as discrepant. Slides were retrieved from our storage area after obtaining institutional IRB approval. A total of three pathologists reviewed and scored all available slides independently using the ASCO/CAP 2007 guidelines. An average score was obtained from the three scores on each case.

The 'old' FDA immunohistochemical stain scoring system:

$0=$ no or incomplete membrane staining in $<10 \%$ of tumor: no overexpression

$1=$ weak, partial membrane staining: no overexpression

$2=$ weak to moderate, complete membrane staining in $>10 \%$ of tumor: indeterminate

$3=$ strong, complete membrane staining in $>10 \%$ of tumor: overexpression

The 'new' 2007 ASCO/CAP guideline scoring system:

$0=$ no staining is observed in invasive tumor cells: no overexpression

$1=$ weak, incomplete membrane staining in any proportion of invasive tumor cells, or weak, complete membrane staining in less than $10 \%$ of cells: no overexpression

$2=$ complete membrane staining that is nonuniform or weak but with obvious circumferential distribution in at least $10 \%$ of cells, or intense complete membrane staining in $30 \%$ or less of tumor cells: equivocal

$3=$ uniform intense membrane staining of more than $30 \%$ of invasive tumor cells: overexpression. 
Table 1 Discordant cases between CNB and EXC

\begin{tabular}{|c|c|c|c|c|}
\hline Case no. & CNB IHC & CNB FISH & $E X C I H C$ & EXC FISH \\
\hline 75 & 1 & 1.3 & 3 & 11.8 \\
\hline 108 & 0 & 1.6 & 3 & 3.7 \\
\hline 9 & 1 & 2.4 & 1 & 0.9 \\
\hline 16 & 1 & 2.4 & 0 & 1.4 \\
\hline 47 & 3 & 2.1 & 2 & 1.6 \\
\hline 52 & 1 & 3 & 1 & 1.0 \\
\hline 68 & 3 & 2.3 & 2 & 1.0 \\
\hline 46 & 3 & 1.4 & 3 & 2.7 \\
\hline 54 & 1 & 1.4 & 1 & 2 \\
\hline 43 & 2 & 4.2 & 2 & 0.9 \\
\hline \multirow[t]{3}{*}{66} & 1 & 1.3 & 3 & 1.5 \\
\hline & FISH amplification & No overexpression or amplification & & \\
\hline & IHC overexpression & & & \\
\hline
\end{tabular}

CNB, core needle biopsy; EXC, excisional biopsy; FISH, fluorescent in situ hybridization; IHC, immunohistochemical stain.

\section{Results}

The total number of specimens evaluated from 125 patients was 260, composed of 129 core biopsies and 131 excisional biopsy specimens. Data analysis was carried out using the criteria before the ASCO/CAP 2007 guidelines for $H E R-2 /$ neu testing.

\section{Discordant Core Needle Biopsy/Excisional Biopsy Data for Fluorescent In Situ Hybridization and Immunohistochemical Stain}

There was complete concordance with respect to both immunohistochemical stain and fluorescent in situ hybridization results for biopsy and excisional biopsy specimens in $87 \%$ of the patients evaluated (109 of 125) (Table 1). Of the remaining $13 \%$ of patients, $2 \%$ (2 of 125) showed discordance in core needle biopsy vs excisional biopsy results, with the immunohistochemical stain and fluorescent in situ hybridization results for the core needle biopsy concurring, yet opposing those of the excisional biopsy specimen; $6 \%$ (8 of 125) showed inconsistencies in only the fluorescent in situ hybridization results between the core needle biopsy and excisional biopsy; 1\% (1 of 125) had only disparate immunohistochemical stain results between the core needle biopsy and excisional biopsy; and 4\% (5 of 125) had concordant fluorescent in situ hybridization results and concordant immunohistochemical stain results, yet the fluorescent in situ hybridization and immunohistochemical stain results opposed each other. Of the 260 specimens evaluated, $5 \%$ (13 of 260) showed inconsistencies between the immunohistochemical stain and fluorescent in situ hybridization results, accounting for 9\% of our patient population (11 of 125). Of these, $4 \%$ (5 of 125) showed neither immunohistochemical stain nor fluorescent in situ hybridization discrepancy, and $5 \%$ (6 of 125) had either immunohistochemical stain or fluorescent in situ hybridization results that did not concur.

Two patients $(2 \%$ of 125) showed complete discordance between their core needle biopsy and excisional biopsy results. For both patients (cases 75 and 108, Table 1), the biopsy immunohistochemical stain showed no overexpression of the HER-2/neu and the fluorescent in situ hybridization analysis similarly showed no amplification, whereas the examination of the excisional biopsy specimens of both cases showed both overexpression by immunohistochemical stain and gene amplification by fluorescent in situ hybridization. Polysomy of chromosome 17 was identified by fluorescent in situ hybridization on the biopsy specimen in one case (case 108). Slide re-review revealed that these two cases show differences in tumor nuclear grade between the core needle biopsy and excisional biopsy, with the excisional biopsy showing more poorly differentiated tumor cells. The inherent small nature of the core needle biopsy may not provide complete representation of the tumor, resulting in discordance due to sampling. Furthermore, the excisional biopsy may show more aggressive population of tumor with the higher nuclear grade, resulting in discordance due to qualitative morphological differences within the same tumor, as seen in cases 75 and 108. Heterogeneity of HER-2/neu status in a tumor sample may occur.

\section{Comparing Fluorescent In Situ Hybridization Data Between Core Needle Biopsy and Excisional Biopsy}

Fluorescent in situ hybridization testing showed $85 \%$ of all specimens to show no HER-2/neu amplification and $15 \%$ to show HER-2/neu gene amplification (see Tables 1 and 2). Of the 125 patients evaluated, 115 (92\%) had fluorescent in situ hybridization core needle biopsy results that were concordant with the corresponding excisional biopsy results. Ten patients ( $8 \%$ of 125 patients) showed inconsistent fluorescent in situ hybridization results between their core needle biopsy and excisional biopsy specimens. Two of these cases (cases 75 and 108), referred to above, show complete discordance of HER-2/neu 
Table 2 FISH concordant and discordant data between core needle and excisional biopsies

\begin{tabular}{|c|c|c|c|c|}
\hline & & & Number of patients & Patients (\%) \\
\hline \multirow[t]{2}{*}{ Concordant } & $92 \%$ & Concordant negative & 102 & $82 \%$ \\
\hline & & Concordant positive & 13 & $10 \%$ \\
\hline \multirow[t]{2}{*}{ Discordant } & $8 \%$ & Discordant: CNB+/EXC- & 6 & $5 \%$ \\
\hline & & Discordant CNB-/EXC+ & 4 & $3 \%$ \\
\hline Total & & & 125 & $100 \%$ \\
\hline
\end{tabular}

CNB, core needle biopsy; EXC, excisional biopsy; FISH, fluorescent in situ hybridization.

immunohistochemical stain and fluorescent in situ hybridization results between the core needle biopsy and excisional biopsy. If these 2 patients are excluded, 8 of the remaining 123 patients, representing $7 \%$ of our patient pool, had discordant fluorescent in situ hybridization results. Six of these cases (5\% of the 123 patients; cases 9 , 16, 43, 47, 52, and 68; Table 1) showed fluorescent in situ hybridization amplification on the core needle biopsy specimen with no HER-2/neu amplification on the excisional specimen. Two cases (2\% of 123 patients, cases 46 and 54; Table 1) showed no gene amplification on core needle biopsy with amplification at the time of tumor excision. These patients fell within the borderline fluorescent in situ hybridization category, one with a core needle biopsy fluorescent in situ hybridization ratio of 2.07 and an excisional biopsy ratio of 1.6 and the other had a core needle biopsy fluorescent in situ hybridization ratio of 1.4 and an excisional biopsy ratio of 2.0. One possible source of discordance could be 'borderline' cases. Before ASCO/CAP 2007 guidelines, a fluorescent in situ hybridization ratio $\geq 2.0$ was scored as amplified according to FDA guidelines. The ASCO/CAP 2007 guidelines for HER-2/neu proposed the category of 'equivocal' for tumors with fluorescent in situ hybridization ratio of 1.8 and 2.2. On the basis of the ASCO/CAP guidelines, we found that our two patients will fall into this 'equivocal' category and may have not received trastuzumab therapy. Interestingly, the corresponding immunohistochemical stain score of one of these equivocal fluorescent in situ hybridization cases was distinctly positive (case 47 with an immunohistochemical stain score of 3) whereas the other was negative (case 54 with an immunohistochemical stain score of 1$)$.

\section{Comparing the Immunohistochemical Stain Data Between Core Biopsy and Excisional Biopsy Specimens}

Immunohistochemical stains performed on the entire set of specimens showed $77 \%$ to be negative for overexpression $(n=199 ; 20 \%$ of all specimens scored as 0 and $57 \%$ scored as 1$), 15 \%$ to be indeterminate for overexpression $(n=40$; immuno-
Table 3 Immunohistochemical stain concordant/discordant data between core needle and excisional biopsies

\begin{tabular}{lrc}
\hline & No. of patients & Patients (\%) \\
\hline Concordant negative & 83 & $66 \%$ \\
Concordant positive & 6 & $5.0 \%$ \\
Indeterminate & 33 & $26 \%$ \\
Discordant CNB+/EXC- & 0 & $0 \%$ \\
Discordant CNB-/EXC+ & 3 & $2 \%$ \\
Total & 125 & $100 \%$ \\
\hline
\end{tabular}

CNB, core needle biopsy; EXC, excisional biopsy.

histochemical stain score as 2), and $8 \%$ to show overexpression $(n=21$; immunohistochemical stain score 3) (Tables 1 and 3).

Of 125 patients, $3(2 \%$, cases 66,75 , and 108) showed discordant immunohistochemical stain results between their core needle biopsy and excisional biopsy specimens. Two of these three cases, described above (cases 75 and 108), show complete discordance with respect to their core needle biopsy and excisional biopsy immunohistochemical stain and fluorescent in situ hybridization data. Excluding these two cases, our data show only $1 \%$ discordant core needle biopsy and excisional biopsy data by immunohistochemical stain. Our data show immunohistochemical stain studies to be more consistent than fluorescent in situ hybridization studies with $99.0 \%$ (122 of 123 patients) and 94.0\% (115 of 123 patients) concordance, respectively.

\section{Comparing the Immunohistochemical Stain and Fluorescent In Situ Hybridization Data}

Of the 260 specimens, the concordance rate of fluorescent in situ hybridization and immunohistochemical stain was 95\% (Tables 4 and 5). Thirteen specimens (5\% discordant) representing 11 patients (9\% of 125 patients) are subdivided as follows: Three of these cases represented overexpression of the HER-2/neu by immunohistochemical stain with no fluorescent in situ hybridization amplification. Ten cases showed no overexpression by immunohistochemical stain with amplification by fluorescent in situ hybridization. Of the three cases that showed immunohistochemical stain overexpression 
without fluorescent in situ hybridization amplification (cases 33, 46, and 66), one showed polysomy of chromosome 17 that was determined at the time of fluorescent in situ hybridization analysis (case 66). No evidence of polysomy was identified in the other two cases. The two other cases showed no identifiable reason for discrepancy.

\section{'Borderline' Cases by Fluorescent In Situ Hybridization}

Five patients showed 'borderline' HER-2/neu amplification on fluorescent in situ hybridization (data shown in Table 6). At the time of fluorescent in situ hybridization interpretation, ratios greater than 2.0 were designated as amplified. Yet, their fluorescent in situ hybridization scores fall within the interval designated as borderline in the 2007 ASCO/CAP guidelines, with a ratio $\geq 1.8$ and $\leq 2.2$. Two of the patients (cases 11 and 78) showed persistent borderline amplification on the core needle biopsy and excisional biopsy specimens with core needle biopsy/excisional biopsy fluorescent in situ hybridization scores of 2.28/2.0 and 2.16/2.04, respectively. Case 70 showed borderline amplification on the core needle biopsy specimen (ratio of 2.2) and definitive amplification on the excisional biopsy specimen (ratio of 2.79). Case 47 showed borderline

Table 4 FISH data on the total number of patients

\begin{tabular}{lccccl}
\hline & FISH+ & FISH- & Total & Total (\%) & \\
\hline 0 & 0 & 52 & 52 & 20.0 & 0 or +1 \\
$1+$ & 10 & 137 & 147 & 57 & $77 \%$ \\
$2+$ & 12 & 28 & 40 & 15 & 5 borderline \\
$3+$ & 18 & 3 & 21 & 8 & 2 borderline \\
Total & 40 & 220 & 260 & & \\
Total (\%) & 15.0 & 85 & 260 & & \\
& \multicolumn{5}{c}{ Discordant } \\
\hline
\end{tabular}

FISH, fluorescent in situ hybridization. fluorescent in situ hybridization amplification on the core needle biopsy specimen (ratio of 2.07) and no amplification on the excisional biopsy specimen (ratio of 1.6). Case 54 showed no fluorescent in situ hybridization amplification on the core needle biopsy specimen (ratio of 1.4) with borderline amplification on the subsequent excisional biopsy specimen (ratio of 2.0).

\section{Effect of Neoadjuvant Chemotherapy}

Five patients received neoadjuvant chemotherapy; all showed no differences in HER-2/neu expression when evaluated by immunohistochemical stain or fluorescent in situ hybridization (data not shown). The one case (case 80) with HER-2/neu amplification by fluorescent in situ hybridization at the time of core needle biopsy showed persistent amplification after receiving chemotherapy at the time of tumor excisional biopsy. The four cases (cases 113, 116, 122, and 124) without HER-2/neu amplification on core needle biopsy continued to not have amplification on excision by fluorescent in situ hybridization.

\section{Immunohistochemical Stain Re-Review of Discrepant Cases}

From 18 patients, 36 were found to have discrepant HER-2/neu results by immunohistochemical stain,

Table 6 Borderline FISH results on core needle biopsy and excision

\begin{tabular}{lcc}
\hline Case no. & CNB FISH ratio & EXC FISH ratio \\
\hline 11 & 2.28 & 2.0 \\
47 & 2.07 & 1.6 \\
54 & 1.4 & 2.0 \\
70 & 2.2 & 2.79 \\
78 & 2.16 & 2.04 \\
\hline
\end{tabular}

CNB, core needle biopsy; EXC, excisional biopsy; FISH, fluorescent in situ hybridization.

Table 5 Discordance between IHC and FISH

\begin{tabular}{|c|c|c|c|c|}
\hline Case no. & CNB IHC & CNB FISH & $E X C I H C$ & EXC FISH \\
\hline 9 & 1 & 2.3 & 1 & 0.98 \\
\hline 13 & 1 & 2.6 & 1 & 2.67 \\
\hline 16 & 1 & 2.41 & 0 & 1.43 \\
\hline 33 & 1 & 0.91 & 2 & 0.94 \\
\hline 40 & 1 & 2.7 & 2 & 2.6 \\
\hline 46 & 3 & 1.36 & 3 & 2.69 \\
\hline 52 & 1 & 3 & 1 & 1.04 \\
\hline 63 & 1 & 2.5 & 1 & 2.53 \\
\hline 66 & 1 & 1.3 & 3 & 1.52 \\
\hline 78 & 2 & 2.16 & 1 & 2.04 \\
\hline \multirow[t]{2}{*}{54} & 1 & 1.4 & 1 & 2 \\
\hline & IHC overexpress, FISH no amplification & IHC no overexpress, FISH amplification & & \\
\hline
\end{tabular}

CNB, core needle biopsy; EXC, excisional biopsy; FISH, fluorescent in situ hybridization; IHC, immunohistochemical stain. 
Table 7 Comparison of Her-2/neu results between pre- and post-ASCO/CAP guidelines

Case no. Average 'new' IHC score Original FISH Original IHC

\begin{tabular}{llcl}
\hline 1 & 1 & 1.56 & 0 \\
2 & 2 & 2.79 & 2 \\
3 & 1 & 1.3 & 1 \\
4 & 1 & 3 & 1 \\
5 & 2 & 1.04 & 1 \\
6 & 2 & 1.3 & 1 \\
7 & 2 & 1.4 & 1 \\
8 & 1 & 2 & 1 \\
9 & 2 & 3.74 & 3 \\
10 & 1 & 2.16 & 2 \\
11 & 1 & 1.03 & 2 \\
12 & 2 & 2.31 & 3 \\
13 & 2 & 1.52 & 3 \\
14 & 2 & 2.07 & 3 \\
15 & 1 & 2.7 & 1 \\
16 & 1 & 1.6 & 2 \\
17 & 2 & 1.36 & 3 \\
18 & 1 & 2.35 & 1 \\
19 & 1 & 0.98 & 1 \\
20 & 1 & 2.28 & 2 \\
21 & 2 & 2 & 2 \\
22 & 1 & 2.6 & 1 \\
23 & 1 & 2.67 & 1 \\
24 & 2 & 2.04 & 1 \\
25 & 0 & 1.43 & 0 \\
26 & 1 & 2.6 & 2 \\
27 & 3 & 11.84 & 3 \\
28 & 3 & 2.53 & 1 \\
29 & 3 & 4.23 & 2 \\
30 & 2 & 0.99 & 2 \\
& 2 & & \\
\hline
\end{tabular}

FISH, fluorescent in situ hybridization; IHC, immunohistochemical stain.

fluorescent in situ hybridization, or both between the core needle biopsy and excisional biopsy specimens and/or to have borderline fluorescent in situ hybridization ratios. Of these cases, 30 of the original immunohistochemical stain slides for HER-2/neu were available for re-review. The 2007 ASCO/CAP criteria for immunohistochemical stain evaluation of HER-2/neu were applied by three separate pathologists. The averages of these scores were taken and compared to the original immunohistochemical stain score (Table 7). The number of discordant immunohistochemical stain/fluorescent in situ hybridization cases is 7 vs 8 when you compare the 2007 ASCO/CAP criteria and the original immunohistochemical stain scores, respectively, which is not statistically different. With 2007 ASCO/CAP criteria score evaluation of HER-2/neu, all seven discrepant cases were undercalled by immunohistochemical stain and showed amplification by fluorescent in situ hybridization. Comparing the original immunohistochemical stain score with the fluorescent in situ hybridization ratios, two of the discrepant cases were overcalled as three on immunohistochemical stain, yet showed no amplification by fluorescent in situ hybridization and six cases were undercalled by immunohistochemical stain. Using the 2007 ASCO/CAP criteria, 12 of the
30 re-reviewed cases would result in reflex fluorescent in situ hybridization testing, whereas using the original FDA-approved immunohistochemical stain score resulted in 9 cases needing reflex fluorescent in situ hybridization.

\section{Discussion}

Determination of HER-2/neu status in breast cancer is important. It is critically important that HER-2/neu studies only be performed on the invasive component of the carcinoma and not on DCIS. DCIS is known to be more positive for HER-2/neu amplification/overexpression than its invasive counterpart in both immunohistochemical stain and fluorescent in situ hybridization tests. Further literature indicates that fluorescent in situ hybridization for HER-2/neu amplification can emerge in atypical ductal hyperplasia and most often appears first in these lesions. These emerging data suggest that amplification of HER-2/neu appears to be mainly involved in initiation of breast oncogenesis. ${ }^{2,3}$

Extensive research has been performed to determine the accuracy of the various testing methods. Of the various methods used to determine HER-2/neu amplification status, immunohistochemical staining and fluorescent in situ hybridization are the two most widely accepted practices. Many pathologists perform the immunohistochemical stain for HER-2/neu receptor expression. Reflexive fluorescent in situ hybridization testing is carried out for a subset of cases with an immunohistochemical stain score of 2 and not for cases with immunohistochemical stain scores of 0,1 , or 3 in most laboratories.

The literature contains many reports on the accuracy of HER-2/neu testing by many different methodologies and new technologies are numerous; ${ }^{4-9}$ however, there is no published study evaluating the concordance of core needle biopsy and excisional biopsy specimen HER-2/neu results from the same patient. Our findings are novel and unique in that similar studies have not been previously published.

Overall, our data support good correlation (87\%) between the immunohistochemical stain and fluorescent in situ hybridization HER-2/neu results of patients' core needle biopsies and excisional specimens. Yet within our population of 125 patients, we also did not obtain perfect concordance between their immunohistochemical stain and fluorescent in situ hybridization results for both core needle biopsy and excisional biopsy.

Amplification of HER-2/neu is reported $10-20 \%$ in literature and our study showed overall 15\% amplification. The discordant rate up to $20 \%$ of HER-2/neu protein expression by immunohistochemical stain and gene amplification by fluorescent in situ hybridization has been documented in several studies. In our study population, there was 
95\% concordance rate between immunohistochemical stain and fluorescent in situ hybridization test.

The possible reasons for inconsistent immunohistochemical stain results are multifactorial: lack of reproducibility of immunohistochemical stain to evaluate HER-2/neu receptor expression with intraobserver and interobserver variability and differences in pre-analytic factors such as fixation time and laboratory-processing variables may have functions in inconsistent results.

Another factor that leads to different HER-2/neu status in both immunohistochemical stain and fluorescent in situ hybridization is the intratumoral heterogeneity due the sampling issue with the core needle biopsy. Two of our cases (cases 75 and 108) showed heterogeneity in tumor nuclear grade; in both, the excisional biopsy shows more poorly differentiated areas than sampled in the core needle biopsy.

Occasional breast cancers with HER-2/neu immunohistochemical stain scores of 3 may be negative for gene amplification by fluorescent in situ hybridization. Polysomy of chromosome 17 has been found as one of the reason for the strong immunohistochemical stain score of 3 and negative amplification by fluorescent in situ hybridization. ${ }^{10-12}$ Downs-Kelly et $a l^{13}$ suggested that polysomy 17 in the absence of HER-2/neu amplification does not have biological influence on HER-2/neu gene expression in breast carcinoma.

We observed no change in HER-2/neu receptor amplification status in the five patients who received neoadjuvant chemotherapy before their excision.

Although, this patient population is quite small, we note that these findings echo the results found in other studies of patients treated with chemotherapy. ${ }^{14}$ However, previous study performed by our group showed changes in the breast biomarkers including HER-2/neu status after neoadjuvant treatment. ${ }^{15}$

In an attempt to resolve some of the discrepancies between the HER-2/neu immunohistochemical stain results of core needle biopsy and excisional biopsy, we re-reviewed 30 discrepant and/or borderline fluorescent in situ hybridization cases applying the new ASCO/CAP 2007 guidelines. Using the 2007 ASCO/CAP criteria, immunohistochemical stain scores of 3 were seen in $17 \%$ of the re-reviewed cases, 2 in $53 \%$, and 0 or 1 in $30 \%$ of cases, whereas re-review utilizing the guidelines before ASCO/CAP showed scores of 3 in $10 \%, 2$ in $40 \%$, and 0 or 1 in $50 \%$. Using 2007 ASCO/CAP guidelines for fluorescent in situ hybridization, there are five cases of a borderline amplification for HER-2/neu.

Upon re-review of the available HER-2/neu slides using the 2007 ASCO/CAP guidelines, there are seven cases with immunohistochemical stain results that were discordant with the fluorescent in situ hybridization results. This is not statistically different from the eight cases with original pre-ASCO/ CAP immunohistochemical stain scores that did not concur with the respective fluorescent in situ hybridization results. We did not find significant changes in terms of resolving the discordant rate of HER-2/neu immunohistochemical stain results with fluorescent in situ hybridization results. Our findings are similar to those of Gilmore et $a l^{16}$ in their abstract presentation in 2008. In addition, our findings are similar to the Brunelli et $a l^{17}$ study that showed the ASCO/CAP scheme has a great concordance coefficient between strong immunohistochemical positivity (scores of 3) and HER-2/neu fluorescent in situ hybridization amplification.

In summary, the most cost-efficient and medically effective way to study breast biomarkers is to do them on the excisional specimen, because the excisional biopsy contains a larger tumor volume to avoid the problem of intratumoral heterogeneity. However, breast biomarkers should be performed on the core needle biopsy of patients who are to undergo neoadjuvant chemotherapy. Although we did not see changes in HER-2/neu status after neoadjuvant treatment in our limited number of cases, other studies have seen such changes; therefore, repeating HER-2/neu studies after neoadjuvant chemotherapy is reasonable. On the basis of our study, we observed that repeating HER-2/neu testing by immunohistochemical stain and/or fluorescent in situ hybridization methods on excisional biopsy is not unreasonable on an individual case, in particular if the excisional biopsy specimen shows different tumor characteristics or different morphology than the core needle biopsy. The HER-2/neu results for both fluorescent in situ hybridization and immunohistochemical stain reflected the tumor grade. If breast biomarkers have been performed on core needle biopsy and show indeterminate/borderline HER-2/neu results by immunohistochemical stain/fluorescent in situ hybridization, we recommend repeating the studies on the excisional biopsy.

\section{Conflict of interest}

The authors declare no conflict of interest.

\section{References}

1 Wolff AC, Hammond ME, Schwartz JN, et al. American Society of Clinical Oncology/College of American Pathologists guideline recommendations for human epidermal growth factor receptor 2 testing in breast cancer. J Clin Oncol 2007;25:118-145.

2 Kobayashi M, Ooi A, Oda Y, et al. Protein overexpression and gene amplification of $c$-erbB-2 in breast carcinomas: a comparative study of immunohistochemistry and fluorescence in situ hybridization of formalin fixed, paraffin-embedded tissues. Hum Pathol 2002;33:21-28.

$3 \mathrm{Xu}$ R, Perle MA, Inghirami G, et al. Amplification of HER-2/neu gene in HER-2/neu-overexpressing and nonexpressing breast carcinomas and their synchronous benign, premalignant, and metastatic lesions detected by fluorescent in-situ hybridization in archival material. Mod Pathol 2002;15:116-124. 
4 Ridolfi RL, Jamehdor MR, Arber JM. HER-2/neu testing in breast carcinoma: a combined immunohistochemical and fluorescence in situ hybridization approach. Mod Pathol 2000;13:866-873.

5 Steven S. Fluorescence in situ hybridization versus immunohistochemistry: importance of clinical outcome. J Clin Oncol 1999;17:3690-3692.

6 Jacobs TW, Gown AM, Yaziji H, et al. Comparison of fluorescence in situ hybridization and immunohistochemistry for the evaluation of HER-2/neu in breast cancer. J Clin Oncol 1999;17:1974-1982.

7 Jacobs TW, Gown AM, Yaziji H, et al. Specificity of HercepTest in determining HER-2/neu status of breast cancers using the United States Food and Drug Administration-approved scoring system. J Clin Oncol 1999;17:1983-1987.

8 Wiley E, Diaz LK. High-quality HER-2 testing: setting a standard for oncologic biomarker assessment. JAMA 2004;291:2019-2020.

9 Gown AM, Goldstein LC, Barry TS, et al. High concordance between immunohistochemistry and fluorescent in situ hybridization testing for HER-2 status in breast cancer requires a normalized immunohistochemical stain scoring system. Mod Pathol 2008;21:1271-1277.

10 Salazar L, Chen L. Impact of polysomy 17 on HER-2/neu immunohistochemistry in breast carcinomas without HER-2/neu gene amplification. J Mol Diagn 2003;5:155-159.

11 Varshney D, Zhou Y, Geller S, et al. Determination of HER-2 status and chromosome 17 polysomy in breast carcinomas comparing HercepTest and PathVysion fluorescent in-situ hybridization assay. Am J Clin Pathol 2004;121:70-77.

12 Hammock L, Lewis M, Phillips C, et al. Strong HER-2/neu protein overexpression by immunohistochemistry often does not predict oncogene amplification by fluorescence in situ hybridization. Hum Pathol 2003;34:1043-1047.

13 Downs-Kelly E, Yoder BJ, Stoler M, et al. The influence of polysomy 17 on HER 2 gene and protein expression in adenocarcinoma of the breast: a fluorescent in situ hybridization, immunohistochemical, and isotopic mRNA in situ hybridization study. Am J Surg Pathol 2005;29:1221-1227.

14 Gong Y, Booser DJ, Sneige N. Comparison of HER-2 status determined by fluorescence in situ hybridization in primary and metastatic breast carcinoma. Cancer 2005;103:1763-1769.

15 Sullivan P, Apple SK. Should histologic type be taken into account when considering neoadjuvant chemotherapy in breast carcinoma? Breast J 2009;15: 146-154.

16 Gilmore HL, Collins LC, Connolly JL, et al. Impact of the new American Society of Clinical Oncology (ASCO)/College of American Pathologists (CAP) guidelines on the determination of HER-2 status. [abstract]. Mod Pathol 2008;21:32 A.

17 Brunelli M, Manfrin E, Martignoni G, et al. HER-2/neu assessment in breast cancer using the original FDA and new ASCO/CAP guideline recommendations: impact on selecting patients for herceptin therapy. Am J Clin Pathol 2008;129:907-911. 